\title{
I Eigentum und Miete: Finanzielle Belastung durch Wohnkosten in Österreich
}

\author{
Wilfried Altzinger ${ }^{\star}$ und Emanuel List**
}

\section{Zusammenfassung}

Die vorliegende Studie präsentiert eine empirische Analyse zu den Wohnkosten österreichischer Haushalte und deren unterschiedliche Belastung für WohnungseigentümerInnen und MieterInnen. EigenheimbesitzerInnen haben im Durchschnitt deutlich höhere Einkommen als Haushalte, die ihren Hauptwohnsitz mieten. Zusätzlich fallen für WohnungseigentümerInnen die Wohnkosten auch absolut niedriger aus, selbst wenn noch ein laufender Kredit abbezahlt wird. Somit ist die relative Wohnkostenbelastung als Anteil am verfügbaren Einkommen für WohnungseigentümerInnen wesentlich geringer als jene für MieterInnen. Folglich führt die Berücksichtigung der Wohnkosten zu einer erhöhten Ungleichheit der (nach Abzug der Wohnkosten) verbleibenden verfügbaren Einkommen. Die Daten zeigen weiters, dass geförderte MieterInnen eine vergleichsweise geringere Wohnkostenbelastung aufweisen als nicht geförderte MieterInnen, und unterstreichen somit die verteilungspolitische Wirksamkeit dieser Politik. Dieser Befund wird auch durch ökonometrische Analysen bestätigt, wobei sich hier zeigt, dass vor allem für junge Haushalte, Singles und Alleinerziehende die Wohnkosten eine deutlich überdurchschnittliche Belastung darstellen. Wohnungspolitische Maßnahmen, die die Belastungen für jene Haushalte senken sollen, die am stärksten von hohen Aufwendungen für das Wohnen betroffen sind, sollten somit in erster Linie bei einer Entlastung von MieterInnen ansetzen.

Schlagwörter: Wohnkosten, Ungleichheit, Einkommensverteilung

Homeowners and Renters: The financial burden of housing costs in Austria

Abstract

This article presents an empirical analysis of the housing costs of Austrian households and the resulting financial burden for homeowners and renters. On average homeowners have significantly higher incomes than households that rent their main residence. In addition, the housing costs for homeowners are also lower in absolute terms, even if a current loan is still being paid off. Thus, the relative housing cost burden as a share of disposable income is much lower for homeowners than for renters. Consequently, the inclusion of housing costs leads to an increased inequality of disposable income (after deducting housing costs). The data also show that subsidized tenants have a comparatively lower housing cost burden than non-subsidized tenants. This finding is also confirmed by econometric exercises, which further show that housing costs represent an above-average burden for young households, singles and especially single parents. Housing policy measures designed to reduce the burden of the households most affected by high housing costs should therefore primarily focus on supporting renters.

Keywords: Housing, Housing Costs, Inequality, Income Distribution

18IIIIIIIIIIIIIIIIIIIIIIIIIIIIIIII

${ }^{*}$ Wilfried Altzinger, Forschungsinstitut Economics of Inequality, Wirtschaftsuniversität Wien

${ }^{*}$ Emanuel List, Forschungsinstitut Economics of Inequality, Wirtschaftsuniversität Wien; Institut für Makroökonomie und Konjunkturforschung, Düsseldorf; emanuel.list@wu.ac.at

Die Studie wurde gefördert durch das Bundesministerium für Soziales, Gesundheit, Pflege und Konsumentenschutz.

2020 | innsbruck university press | Innsbruck

Momentum Quarterly I ISSN 2226-5538 I momentum-quarterly.org

Vol. 9, No 3 I DOI 10.15203/momentumquarterly.vol9.no3.p161-178

Beiträge in Momentum Quarterly stehen unter der Lizenz Creative Commons Namensnennung 4.0 International. 


\section{Einleitung}

Die Wohnsituation in Österreich zeigt sich im europäischen Vergleich sehr differenziert: einerseits ist der Anteil an MieterInnen mit über 40 Prozent nach Deutschland der höchste; zusätzlich ist der Anteil an sozialem Wohnbau und geförderten Mieten besonders stark ausgeprägt (Kunnert/Baumgartner 2012) Die wirtschaftspolitischen Maßnahmen umfassen beispielsweise subventionierte Kredite (Wohnbauförderung) oder gemeinnützigen Wohnbau nach dem Wohnungsgemeinnützigkeitsgesetz. Laut diesem Gesetz dürfen gemeinnützige Wohnbauträger nur eine Miete verlangen, die kostendeckend ist. Des Weiteren gibt es beispielsweise Höchstgrenzen für die Gehälter der MitarbeiterInnen dieser Wohnbauträger. Darüber hinaus müssen die Gewinne in österreichische Wohnprojekte reinvestiert werden. Daneben gibt es noch das Mietrechtsgesetz, das vor allem die Miethöhe für Objekte, die vor 1945 erbaut wurden, begrenzt. Die vierte Säule der Wohnpolitik umfasst Gemeindebauwohnungen, wobei hier Wien den Großteil der zur Verfügung gestellten Objekte verwaltet. Diese Wohnungen werden deutlich unter dem Marktpreis vermietet.

Durch diesen Mix aus Maßnahmen bleibt der Mietaufwand in Österreich im europäischen Vergleich verhältnismäßig gering (Kunnert/Baumgartner 2012), während gleichzeitig die hohen Einkommensschwellen für gemeinnützige Wohnungen soziale Segregation und eine Stigmatisierung des öffentlichen Wohnungsbaus verhindern (Fessler/Rehm/Tockner 2016).

Dennoch gibt es aufgrund des Immobilienbesitzes strukturelle Unterschiede für die ökonomischen Lebensrealitäten, die vor allem durch den Anstieg der Immobilien- und Mietpreise der vergangenen Jahre verstärkt wurden. Wenn zwei Haushalte verglichen werden, die bezüglich Einkommen, Wohnverhältnisse etc. die gleichen Charakteristika aufweisen, jedoch der eine die Immobilie, in dem die Mitglieder wohnen, besitzt und der andere einen wesentlichen Teil des Haushaltseinkommens für Miete ausgibt, wäre es nicht plausibel, deren ökonomische Situation gleich zu bewerten. Um eine umfassende Analyse der Einkommen anzustellen und für die zusätzlichen Konsummöglichkeiten zu korrigieren, wird daher empfohlen, nicht-monetäre Einkommensflüsse aus Immobilienbesitz zu imputieren (International Labour Organisation 2003; Canberra Group 2011; Organization for Economic Cooperation and Development 2013). In mehreren wissenschaftlichen Arbeiten wurde bereits festgehalten, dass es bezüglich der nicht-monetären Einkommen aus Immobilienbesitz, den sogenannten imputierten Mieten, zu einer steuerlichen Ungleichbehandlung zugunsten der EigenheimbesitzerInnen kommt (Figari/Paulus et al. 2017; Fessler/Schürz et al. 2010).

Betrachtet man den Sachverhalt von der Kostenseite, zeigt sich ein ähnliches Bild, da EigenheimbesitzerInnen keine Miete zahlen und somit die gesamten Wohnkosten tendenziell niedriger ausfallen werden. Aber auch wenn ImmobilienbesitzerInnen keine Mieten bezahlen, müssen diese ebenso wie die MieterInnen Betriebskosten oder Instandhaltungskosten und bei laufenden Hypotheken auch Zins- und Tilgungszahlungen leisten.

Die Größenordnung der Wohnkosten der einzelnen Gruppen ist also ex ante nicht definierbar. In dieser Auswertung soll deshalb analysiert werden, wie sich die Belastung durch Wohnkosten auf die Einkommen und deren Verteilung auswirken. Somit soll eine empirische Grundlage gebildet werden, um zielgerichtete wirtschafts- bzw. wohnungspolitische Maßnahmen $\mathrm{zu}$ evaluieren, die eine soziale Verträglichkeit der Wohnkosten garantieren und einer Segregation der Wohngruppen vor dem Hintergrund von steigenden Miet- und Immobilienpreisen entgegenwirken.

\section{Literatur}

Für Österreich gibt es zwar mehrere Untersuchungen zur Wohnkostenbelastung, jedoch zumeist mit unterschiedlicher Datengrundlage, wodurch direkte Vergleiche nur eingeschränkt möglich sind. Die meisten diesbezüglichen Untersuchungen stammen dabei von AutorInnen aus der Oesterreichischen Nationalbank (OeNB) (Beer/Wagner 2012; Beer/Wagner 2017; Wagner 2011).

Die für unsere Zwecke wichtigste Studie ist jene von Beer/Wagner (2017), die auf Grundlage der Daten des Household, Finance and Consumption Survey (HFCS) der Europäischen Zentralbank für 2014 einen Vergleich der Ausgaben für Wohnkosten für die Länder Österreich, Deutschland sowie Italien erstellt. Ohne hierbei auf die genaue Definition von Wohnkosten sowie verfügbarem Einkommen einzugehen, wollen wir die für uns wichtigsten Ergebnisse zusammenfassen (s. Tabelle 1). Für Österreich zeigt sich für 2014 eine durchschnittliche Wohnkostenbelastung von 28,6 Prozent (Median 25,6 Prozent). Um die unterschiedliche Wohnkostenbelastung nach Haushaltstyp zu unterscheiden, wurde 


\begin{tabular}{|c|c|c|c|}
\hline HFCS 2014 & Mittelwert & Median & in Relation zum Median \\
\hline Insgesamt & 28,6 & 25,6 & $100 \%$ \\
\hline Eigentümerlnnen (E.) & 20,7 & 17,6 & $69 \%$ \\
\hline E. ohne Kredit & 17,3 & 15,6 & $61 \%$ \\
\hline E. mit Kredit & 29,4 & 26,0 & $102 \%$ \\
\hline Mieterlnnen & 39,2 & 37,0 & $145 \%$ \\
\hline Freie Nutzerlnnen & 14,1 & 11,7 & $46 \%$ \\
\hline \multicolumn{4}{|l|}{ Einkommensquartil } \\
\hline 1 & 41,3 & 39,9 & $156 \%$ \\
\hline 2 & 30,2 & 28,7 & $112 \%$ \\
\hline 3 & 24,8 & 22,6 & $88 \%$ \\
\hline 4 & 18,1 & 15,7 & $61 \%$ \\
\hline \multicolumn{4}{|l|}{ Vermögensquartil } \\
\hline 1 & 40,6 & 39,8 & $155 \%$ \\
\hline 2 & 31,4 & 29,9 & $117 \%$ \\
\hline 3 & 23,3 & 20,1 & $79 \%$ \\
\hline 4 & 19,2 & 16,4 & $64 \%$ \\
\hline
\end{tabular}

Quelle: Beer/Wagner (2017), Tabelle 2, S. 55, eigene Berechnungen

der Wert des Medians auf 100 normiert. Dabei zeigt sich klar, dass MieterInnen mit einem Wert von 145 im Durchschnitt eine wesentlich höhere Wohnkostenbelastung aufweisen als EigentümerInnen (ohne Kredite: 61; mit Krediten: 102). Die mit Abstand geringste Wohnkostenbelastung weisen die sogenannten ,Freien NutzerInnen' auf, die zumeist eine Wohnung von ihren Eltern unentgeltlich zur Verfügung gestellt bekommen (s. dazu Kap. 3). Darüber hinaus zeigt sich in den Daten eine klare Hierarchie hinsichtlich den Einkommensund Vermögensverhältnissen: Haushalte mit hohem Einkommen sowie Haushalte mit hohem Vermögen haben eine wesentlich geringere Wohnkostenbelastung als Haushalte am unteren Ende der Einkommens- sowie Vermögensverteilung. Die Wohnkostenbelastung des 1. Quartils macht in beiden Fällen das 2,5-fache des 4 . Quartils aus.

Die Berechnungen von Beer und Wagner (2012) beruhen hingegen auf Daten einer eigenen Befragung, die die OeNB gemeinsam mit dem Institut für empiri- sche Sozialforschung (IFES) im Frühjahr 2012 durchgeführt hat. Zwar zeigt sich auch in dieser Studie eine klare Wohnkostenbelastungshierarchie hinsichtlich der Einkommen, und zwar sowohl bei MieterInnen als auch bei EigentümerInnen, jedoch ist die Wohnkostenbelastung mit 29,1 \% für MieterInnen (gegenüber 37,0 \% im HFCS 2014) sowie 19,0 \% für EigentümerInnen (gegenüber 17,6 \% im HFCS 2014) wesentlich geringer, als die Daten des HFCS anzeigen.

Die Studie von Wagner verwendet Daten aus eine Immobilienerhebung der OeNB von 2008 (Wagner 2011). Auch hier zeigt sich, dass höhere Einkommen (sowohl bei den MieterInnen als auch bei den EigentümerInnen) eine geringe Wohnkostenbelastung aufweisen. Die Wohnkostenbelastung für MieterInnen beträgt in dieser Untersuchung jedoch nur 24,1\%, während jene für EigentümerInnen sogar mit nur 5,9 \% ausgewiesen wird. Der Unterschied zwischen MieterInnen und EigentümerInnen ist somit enorm. In dieser Studie wird die Wohnkostenbelastung auch hinsichtlich eini- 
Tabelle 2: Subjektive sowie objektive Wohnkostenbelastung

\begin{tabular}{|c|c|c|c|c|c|c|}
\hline & $\begin{array}{c}\text { (1) } \\
\text { keine } \\
\text { Belastung }\end{array}$ & $\begin{array}{c}\text { (2) } \\
\text { gewisse } \\
\text { Belastung }\end{array}$ & $\begin{array}{c}\text { (3) } \\
\text { starke } \\
\text { Belastung }\end{array}$ & $\begin{array}{c}\text { (4) } \\
\text { subjektive } \\
\text { Belastung }\end{array}$ & $\begin{array}{c}\text { (5) } \\
\text { Wohnkosten- } \\
\text { belastung (in \%) } \\
\text { (obj. Belastung) }\end{array}$ & $\begin{array}{c}\text { (6) } \\
\text { subjektive/ } \\
\text { objektive Belas- } \\
\text { tung (normiert) }\end{array}$ \\
\hline & & in $\%$ & & & & \\
\hline Insgesamt & 39 & 50 & 11 & 1,72 & 21 & 100 \\
\hline \multicolumn{7}{|l|}{ Einkommensgruppen } \\
\hline niedrig $(<60 \%)$ & 29 & 45 & 26 & 1,97 & 45 & 53 \\
\hline mittel (60 \% bis $180 \%)$ & 39 & 52 & 9 & 1,70 & 17 & 122 \\
\hline $\operatorname{hoch}(>180 \%)$ & 58 & 40 & 2 & 1,44 & 8 & 220 \\
\hline \multicolumn{7}{|l|}{ Rechtsverhältnis } \\
\hline Hauseigentum & 38 & 54 & 8 & 1,70 & 14 & 148 \\
\hline Wohnungseigentum & 50 & 45 & 5 & 1,55 & 17 & 111 \\
\hline Gemeindewohnung & 29 & 50 & 21 & 1,92 & 27 & 87 \\
\hline Genossenschaftswohnung & 37 & 48 & 15 & 1,78 & 27 & 80 \\
\hline Andere Hauptmiete & 39 & 47 & 14 & 1,75 & 33 & 65 \\
\hline Sonstige & 45 & 47 & 8 & 1,63 & 15 & 133 \\
\hline
\end{tabular}

Quelle: Statistik Austria (2020); EU-SILC 2019, Tab. 2.16, eigene Berechnungen

ger individuellen Charakteristika untersucht. Dabei zeigt sich u. a., dass die Wohnkostenbelastung mit dem Alter des Haushaltsvorstandes für EigentümerInnen deutlich sinkt, während jene für MieterInnen bis zum Alter von etwa 60 Jahren leicht sinkt und danach nochmals deutlich ansteigt (Wagner 2011: 141f.). Die Erklärung für diese unterschiedlichen Verläufe ist bedingt durch zwei Entwicklungen: Einerseits ist bei älteren Eigentümerhaushalten die Höhe der noch aushaftenden Darlehen schon geringer als bei jüngeren bzw. das Darlehen ist bereits zurückgezahlt; die Mietkosten sind jedoch relativ unabhängig vom Lebensalter. Andererseits steigt aber das verfügbare Einkommen mit dem Lebensalter für beide Wohntypen, allerdings nur bis zum Eintritt in die Pension. Somit weisen WohnungseigentümerInnen über das Lebensalter einen kontinuierlichen Rückgang der Wohnkostenbelastung auf, während dieser bei den MieterInnen nur relativ gering ist, aber mit Pensionsbeginn wieder steigt. Altersarmut wird somit bei MieterInnen sicherlich häufiger anzutreffen sein als bei WohnungseigentümerInnen.

Was können wir aus den Ergebnissen dieser Untersuchungen lernen? Der zentrale Grund für die unterschiedlichen Belastungsquoten zwischen diesen
Studien liegt vor allem in der Definition von ,Wohnkosten' sowie, verfügbarem Einkommen' (Beer/Wagner 2012: 84ff.; Beer/Wagner 2017: 48ff.; Wagner 2011: 140f.). Generell ist bei einem Vergleich von Studien mit unterschiedlicher Datengrundlage immer Vorsicht geboten. Wichtiger hingegen ist die Frage, ob strukturelle Muster über die Studien hinweg erkennbar sind. Und dabei zeigen sich einheitlich zumindest immer zwei Muster: Erstens: Die Wohnkostenbelastung von MieterInnen ist immer (wesentlich) höher als jene von EigentümerInnen. Zweitens: Die Wohnkostenbelastung von Haushalten mit hohem Einkommen bzw. hohem Vermögen ist immer niedriger als die von Haushalten mit niedrigem Einkommen bzw. Vermögen. Selbstverständlich sind diese beiden Beobachtungen Ergebnis ein und desselben Musters: Haushalte mit niedrigem/hohem Einkommen sind zu einem wesentlich höheren/niedrigeren Anteil Mieter als Eigentümer.

Neben der Wohnkostenbelastung generell ist es auch interessant, einen detaillierten Blick auf den Unterschied zwischen objektiver und subjektiver Wohnkostenbelastung zu werfen. Dabei geht es um die Frage, wie die Wohnkostenbelastung individuell wahrgenommen wird und aufgrund welcher Krite- 
rien eine unterschiedliche Wahrnehmung erfolgt. Amann und Mundt (2019: 24) stellen dazu zwar fest, dass „die objektive und die subjektiv empfundene Belastung dabei nur teilweise in dieselbe Richtung gehen“, untersuchen diese Frage jedoch nicht weiter. Der Tabellenband der Statistik Austria (2020) zur EUSILC-Befragung 2019 bietet uns zu dieser Frage erste Hinweise. In einer eigenen Tabelle (Tabelle 2.16) sind dabei die subjektive Wohnkostenbelastung sowie die gesamten Wohnkosten nach soziodemografischen Merkmalen auf Haushaltsebene aufgelistet (s. Tabelle 2). Die subjektive Belastung wird dabei abgefragt nach ,keine', ,gewisse sowie ,starke‘ Belastung (Spalten (1) bis (3)); die objektive Wohnkostenbelastung wird gemessen als Anteil der Wohnkosten am gesamten Haushaltseinkommen (Spalte (5)). Um die subjektive Belastung quantifizieren zu können, haben wir den Antworten Werte zugeordnet: 1 für ,keine` Belastung, 2 für ,gewisse` Belastung sowie 3 für ,starke` Belastung. Diese Werte wurden entsprechend ihrer Anteilswerte gewichtet, womit sich ein Indikator für die subjektive Wohnkostenbelastung errechnet, der Werte zwischen 1 und 3 annehmen kann (Spalte (4)). Sodann wurde durch einfache Division von subjektiver und objektiver Belastung ein Indikator gebildet, dessen Wert für alle Haushalte auf 100 normiert wurde (Spalte (6)). Werte über 100 weisen auf eine individuelle Überschätzung der tatsächlichen Wohnungskostenbelastung hin, während Werte von unter 100 eine individuelle Unterschätzung anzeigen.

Die Ergebnisse dieser Berechnungen geben uns dabei einen ersten Hinweis, dass Haushalte mit niedrigen Einkommen ihre tatsächliche Belastung eher unterschätzen, während Haushalte mit hohen Einkommen ihre objektive Belastung generell überschätzen. Auch hinsichtlich der Rechtsverhältnisse der Haushalte zeigt sich ein klares Ergebnis: Während insbesondere Haus-, aber auch WohnungseigentümerInnen ihre Wohnkostenbelastung generell überschätzen, zeigt sich sowohl für geförderte als auch für nicht geförderte MieterInnen generell eine Unterschätzung der Belastung. In Kapitel 6 der vorliegenden Studie wird dieser Aspekt noch detaillierter untersucht.

\section{Daten und Wohntypologisierung}

In dieser Ausarbeitung werden die Wohnkosten für verschiedene Wohntypen und deren Anteil am Einkommen analysiert. Die verwendeten Daten stammen aus dem EU-SILC-Datensatz 2017 (European Union
Statistics on Income and Living Conditions). SILC ist eine Erhebung, bei der jährlich soziodemografische Informationen von privaten Haushalten der Europäischen Union durch die nationalen Statistikinstitute erhoben und aufbereitet werden. Da die Befragung unter anderem auf die Einkommens- und Wohnsituation abzielt, sind die Daten eine geeignete Basis für die Fragestellung in diesem Artikel. Folgende Wohntypen werden dabei definiert:

- Eigenheimbesitzer mit Kredit: Haushalte, die ihren Hauptwohnsitz besitzen und eine offene Hypothek bedienen müssen.

- Eigenheimbesitzer ohne Kredit: Haushalte, die ihren Hauptwohnsitz besitzen, keine Hypothek.

- Mieter (nicht gefördert): Haushalte, die für ihren Hauptwohnsitz Miete bezahlen.

- Mieter (gefördert): Haushalte, die für ihren Hauptwohnsitz aufgrund von sozialem Wohnbau etc. einen reduzierten Mietbetrag zahlen.

- Freie Nutzer: Haushalte, die ihren Hauptwohnsitz kostenlos zur Verfügung gestellt bekommen. Dabei handelt es sich zumeist um Erwachsene, die in einer Immobilie der Eltern wohnen, aber einen eigenen Haushalt führen, oder auch Eltern, die den Kindern die Immobilie bereits überschrieben haben, aber noch dort wohnen.

- Eigenheimbesitzer: Alle EigenheimbesitzerInnen

- Mieter: Alle MieterInnen

- Alle Haushalte: Alle aufgelisteten Wohntypen

In Abbildung 1 werden die Wohnkosten für die verschiedenen Wohntypen grafisch dargestellt. Daraus ergeben sich folgende (monatliche) Wohnkosten für die einzelnen Gruppen:

\section{- Wohnkosten}

Für MieterInnen: Miete, Dienstleistungen und Gebühren (Abwasserentsorgung, Müllabfuhr etc.) (falls von den MieterInnen bezahlt), Steuern auf die Immobilie (falls zutreffend), regelmäßige Wartung und Instandhaltung, Kosten der Versorgungseinrichtungen (Wasser, Strom, Gas und Heizung).

Für EigentümerInnen: laufende Zinszahlungen für den Immobilienkredit, Dienstleistungen und Gebühren (Abwasserentsorgung, Müllabfuhr etc.), Steuern auf die Immobilie (falls zutreffend), regelmäßige Wartung und Instandhaltung, Kosten der Versorgungseinrichtungen (Wasser, Strom, Gas und Heizung).

\section{- Wohnkosten + Tilgung}

Diese Position ist nur für EigentümerInnen mit Kredit definiert und setzt sich aus den Wohnkosten und den laufenden Tilgungszahlungen zusammen. QUARTERLY "IIIIIIIIIIIIIIIII

165 


\section{Gesamte Wohnkosten}

(EU-SILC)

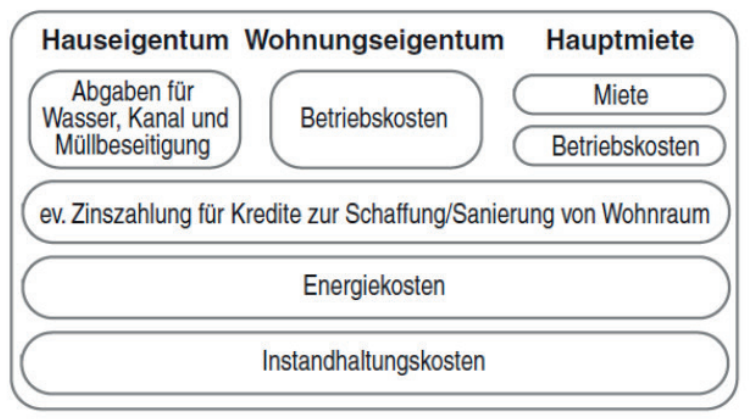

Quelle: Statistik Austria 2020

Abbildung 2: Wohntypen entlang der Verteilung des verfügbaren Einkommens (in Prozent)

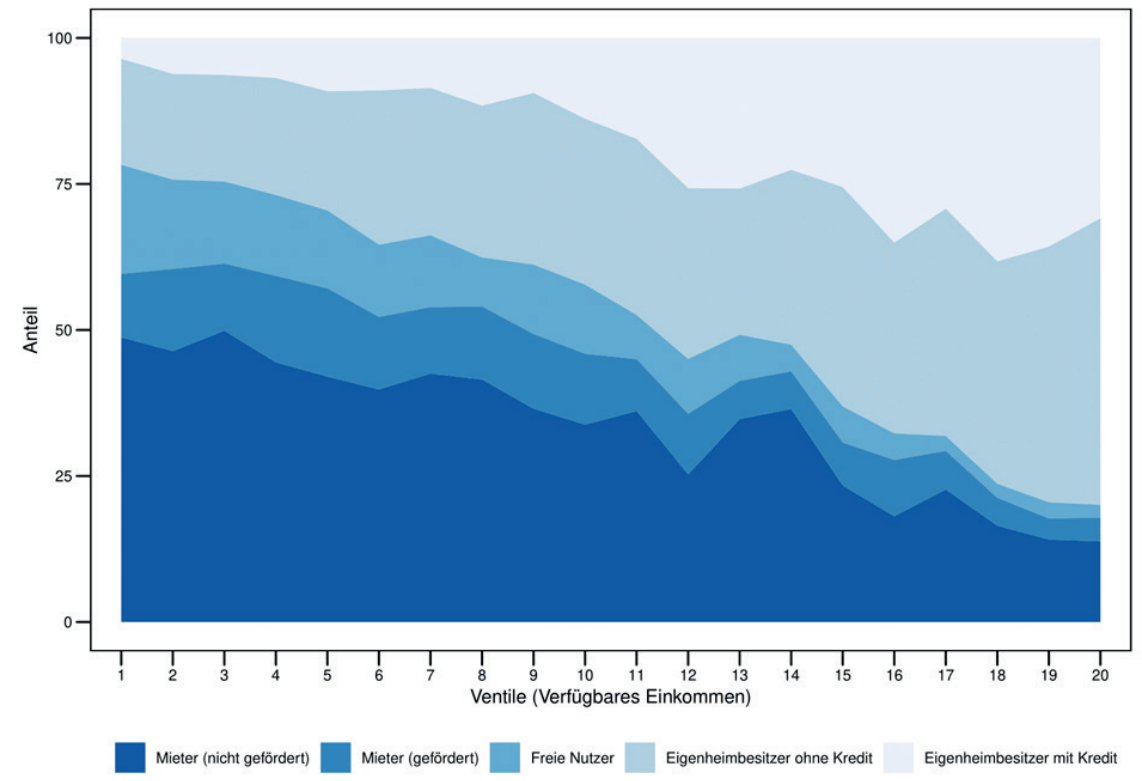

Quelle: SILC 2017

Das folgende Einkommenskonzept wird ausgehend vom Jahresgehalt (inkl. Sonderzahlungen) ebenso wie die Wohnkosten - pro Monat betrachtet:

Verfügbares Einkommen

Sämtliche Einkommen und Sozialleistungen, die dem Haushalt nach Abzug von Steuern und Abgaben zur Verfügung stehen.

\section{Allgemeine Ergebnisse}

In diesem Kapitel werden die berechneten Wohnkosten zuerst allgemein und anschließend über die gesamte Einkommensverteilung als Anteil am verfügbaren Einkommen dargestellt und diskutiert. In Österreich zahlen ca. 43 Prozent der Bevölkerung Miete für ihren Hauptwohnsitz (siehe Tabelle 1). Dabei machen wiederum MieterInnen, die am privaten Markt mieten, 33 Prozent und Haushalte, die in geförderten Mietwohnungen leben, 10 Prozent aus. Der restliche Anteil setzt sich aus 29 Prozent EigentümerInnen ohne Hypothek, 19 Prozent EigentümerInnen mit Hypothek und 9 Prozent Freien NutzerInnen zusammen.

In Abbildung 2 ist $\mathrm{zu}$ sehen, dass diese Anteile entlang der Einkommensverteilung nicht konstant 
Tabelle 3: Durchschnittliche monatliche Wohnkosten absolut in Euro und als Anteil am verfügbaren Einkommen (in Prozent)

\begin{tabular}{|c|c|c|c|c|c|c|c|c|}
\hline & \multirow{2}{*}{$\begin{array}{l}\text { Verfügbares } \\
\text { Einkommen }\end{array}$} & \multicolumn{2}{|c|}{ Wohnkosten } & \multicolumn{2}{|c|}{$\begin{array}{l}\text { Wohnkosten + } \\
\text { Tilgung }\end{array}$} & \multicolumn{2}{|c|}{ Haushaltsmitglieder } & \multirow{2}{*}{$\begin{array}{l}\text { Anteil } \\
\text { in } \%\end{array}$} \\
\hline & & absolut & in $\%$ & absolut & in $\%$ & gesamt & erwerbstätig & \\
\hline Eigenheimbesitzer & 4419 & 448 & 10,1 & - & - & 3,0 & 1,7 & 47,8 \\
\hline davon Eigenheimbesitzer mit Kredit & 4733 & 532 & 11,2 & 916 & 19,4 & 3,1 & 2,2 & 18,5 \\
\hline Freie Nutzer & 2524 & 204 & 8,1 & - & - & 2,2 & 0,4 & 9,1 \\
\hline Mieter & 2877 & 646 & 22,4 & - & - & 2,3 & 1,3 & 43,1 \\
\hline davon Mieter (gefördert) & 2870 & 600 & 20,9 & - & - & 2,2 & 1,4 & 9,8 \\
\hline davon Mieter (nicht gefördert) & 2879 & 659 & 22,9 & - & - & 2,3 & 1,3 & 33,3 \\
\hline
\end{tabular}

Quelle: SILC 2017

Abbildung 3: Wohntypen entlang der Verteilung des verfügbaren Einkommens nach Abzug der Wohnkosten (in Prozent)

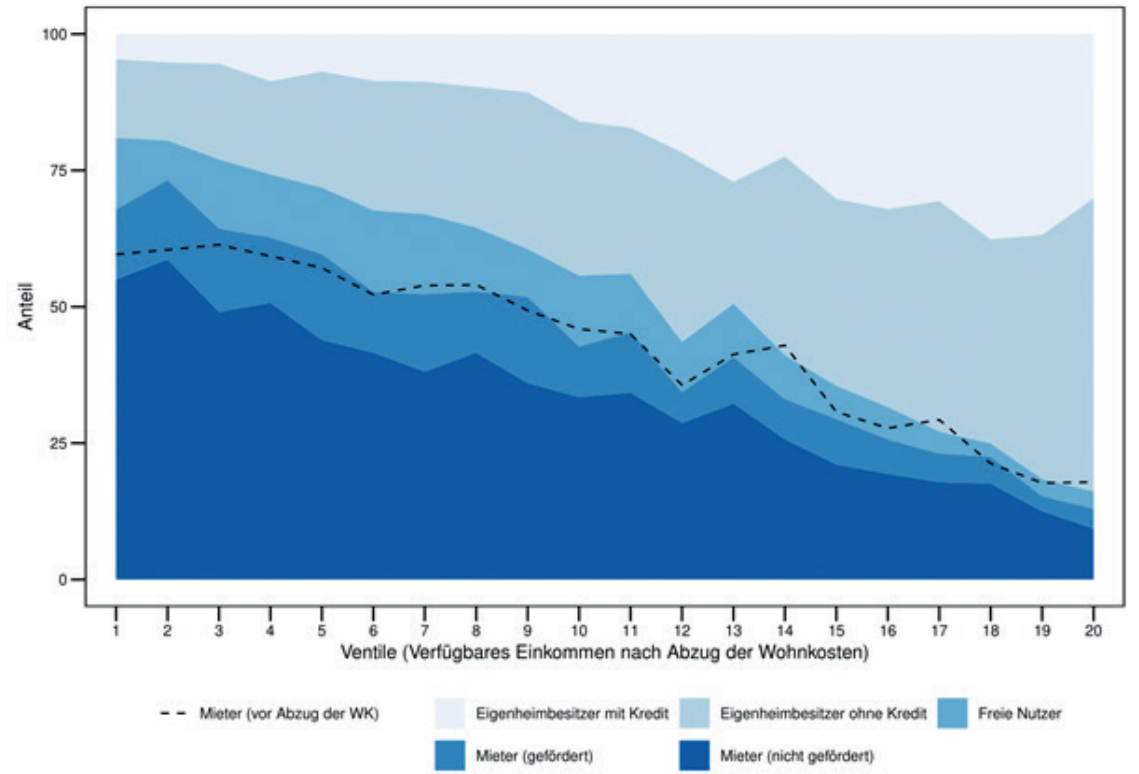

Quelle: SILC 2017

sind, sondern jener der EigentümerInnen mit steigendem Einkommen zunimmt. Haushalte, die sich also am unteren Ende der Einkommensverteilung befinden, haben eine höhere Wahrscheinlichkeit, für ihren Hauptwohnsitz Miete zu bezahlen. Auf der anderen Seite sind es zumeist Haushalte mit Wohnungseigentum, die am oberen Ende der Einkommensverteilung $\mathrm{zu}$ finden sind. Vor allem EigentümerInnen mit laufendem Kredit sind besonders häufig Haushalte mit einem hohen Einkommen. Dieser Umstand ergibt sich dadurch, dass für die Aufnahme eines Wohnkredites ein gewisses Einkommensniveau nötig ist.
Freie NutzerInnen befinden sich tendenziell am unteren Ende der Einkommensverteilung. Bei diesen Haushalten handelt es sich oftmals um junge Erwachsene, die im Elternhaus wohnen, oder auch ältere Personen, die ihren Kindern bereits das Immobilieneigentum überschrieben haben. $\mathrm{Da}$ in diesen Lebensphasen (Ausbildung, Karrierebeginn oder Pension) die Erwerbstätigkeit geringer ist und das Einkommen tendenziell niedriger ausfällt, ist dies wenig überraschend. Bereits vor dem Miteinbeziehen von Wohnkosten lässt sich also eine deutliche Segregation anhand der Wohntypen feststellen, da MieterInnen 
Tabelle 4: Wohnkostenanteile am verfügbaren Einkommen nach Gruppen

\begin{tabular}{|c|c|c|c|c|}
\hline \multicolumn{5}{|c|}{ Im einkommensschwächsten Viertel verändern sich die Anteile wie folgt: } \\
\hline & vor Abzug der WK & $\rightarrow$ & nach Abzug der WK & Gesamtanteil \\
\hline Mieter (nicht gefördert): & $46,3 \%$ & $\rightarrow$ & $51,5 \%$ & $33,3 \%$ \\
\hline Mieter (gefördert): & $13,3 \%$ & $\rightarrow$ & $14,1 \%$ & $9,8 \%$ \\
\hline Eigenheimbesitzer ohne Kredit: & $19,0 \%$ & $\rightarrow$ & $16,9 \%$ & $29,2 \%$ \\
\hline Eigenheimbesitzer mit Kredit: & $6,4 \%$ & $\rightarrow$ & $6,2 \%$ & $18,5 \%$ \\
\hline \multicolumn{5}{|c|}{ Im einkommensstärksten Viertel ergeben sich folgende Zusammensetzungen der Anteile: } \\
\hline & vor Abzug der WK & $\rightarrow$ & nach Abzug der WK & Gesamtanteil \\
\hline Mieter (nicht gefördert): & $17,0 \%$ & $\rightarrow$ & $15,3 \%$ & $33,3 \%$ \\
\hline Mieter (gefördert): & $5,8 \%$ & $\rightarrow$ & $4,6 \%$ & $9,8 \%$ \\
\hline Eigenheimbesitzer ohne Kredit: & $40,5 \%$ & $\rightarrow$ & $42,9 \%$ & $29,2 \%$ \\
\hline Eigenheimbesitzer mit Kredit: & $33,8 \%$ & $\rightarrow$ & $33,5 \%$ & $18,5 \%$ \\
\hline
\end{tabular}

Quelle: SILC 2017. Die Spalte ,Gesamtanteil` entspricht dem durchschnittlichen Anteil über alle Einkommensgruppen

überproportional oft in den unteren Einkommenssegmenten zu finden sind.

Wie in Tabelle $3 \mathrm{zu}$ sehen ist, betragen die durchschnittlichen Wohnkosten per Haushalt und Monat ca. 511 Euro und machen damit rund 14 Prozent des mittleren verfügbaren Einkommens aus. Für geförderte und nicht geförderte MieterInnen ist dieser Anteil mit 21 Prozent bzw. 23 Prozent deutlich höher. Dies ergibt sich zum einen daher, dass die gesamten absoluten Wohnkosten für MieterInnen höher sind (6oo Euro bzw. 659 Euro), aber auch die verfügbaren Einkommen geringer ausfallen (2.87o Euro bzw. 2.879 Euro) als jene der EigenheimbesitzerInnen. EigentümerInnen haben im Durchschnitt mehr erwerbstätige Haushaltsmitglieder und deutlich höhere Einkommen, wobei jenes der Haushalte mit Hypotheken mit durchschnittlich 4.733 Euro noch einmal deutlich über jenem der EigentümerInnen ohne Hypothek (4.220 Euro) liegt. Da Tilgungszahlungen ein Investment darstellen, werden nur die Zinszahlungen zu den Wohnkosten gezählt, nicht aber die Tilgungszahlungen selbst. Interessant ist dennoch, dass für EigentümerInnen mit Kredit die zusammengezählte Belastung aus Wohnkosten und Tilgungszahlungen 19,4 Prozent des verfügbaren Einkommens ausmacht und damit noch immer unter der Wohnkostenbelastung (WKB) der MieterInnen liegt.

Nach Abzug der Wohnkosten (WK) vom verfügbaren Einkommen verstärkt sich die Segregation anhand der Wohntypen nochmals deutlich (siehe Abbildung 3). Die gestrichelte Linie kennzeichnet den Anteil der
MieterInnen (gefördert + nicht gefördert) vor Abzug der WK. Hier wird ersichtlich, dass deren Anteil in den unteren Einkommensschichten deutlich ansteigt.

\subsection{Wohnkosten als Anteil am verfügbaren Einkommen}

In diesem Abschnitt werden die Wohnkosten als Anteil am verfügbaren Einkommen nun über die gesamte Einkommensverteilung dargestellt. Dies erlaubt eine detaillierte Analyse, da man sehen kann, wie stark die einzelnen Wohntypen an den Rändern der Verteilung von Wohnkosten betroffen sind.

Abbildung 4 zeigt den Anteil der Haushalte, die mehr als X Prozent ihres verfügbaren Einkommens für die Wohnkosten aufwenden. So geben beispielsweise 25 Prozent aller Haushalte mehr als 25,3 Prozent ihres verfügbaren Einkommens für Wohnkosten aus, während es bei den MieterInnen 35,5 Prozent und bei den EigenheimbesitzerInnen nur 16,1 Prozent sind. Der Wohnungskostenanteil der MieterInnen ist über den gesamten Verlauf deutlich höher, wobei Belastungen von über 50 Prozent beinahe ausschließlich bei MieterInnen auszumachen sind. Ein Viertel aller Haushalte wendet nicht mehr als 9,3 Prozent des verfügbaren Einkommens für Wohnkosten auf. Bei den EigenheimbesitzerInnen beträgt dieser Wert 7,5 Prozent. Die 25 Prozent der MieterInnen mit den niedrigsten Ausgaben für Wohnkosten geben zumindest 17,3 Prozent aus und haben damit eine mehr als doppelt so hohe Wohnkostenbelastung wie Haushalte mit Wohnungseigentum. 
Abbildung 4: Anteil der Haushalte, die mehr als X Prozent ihres verfügbaren Einkommens für Wohnkosten ausgeben

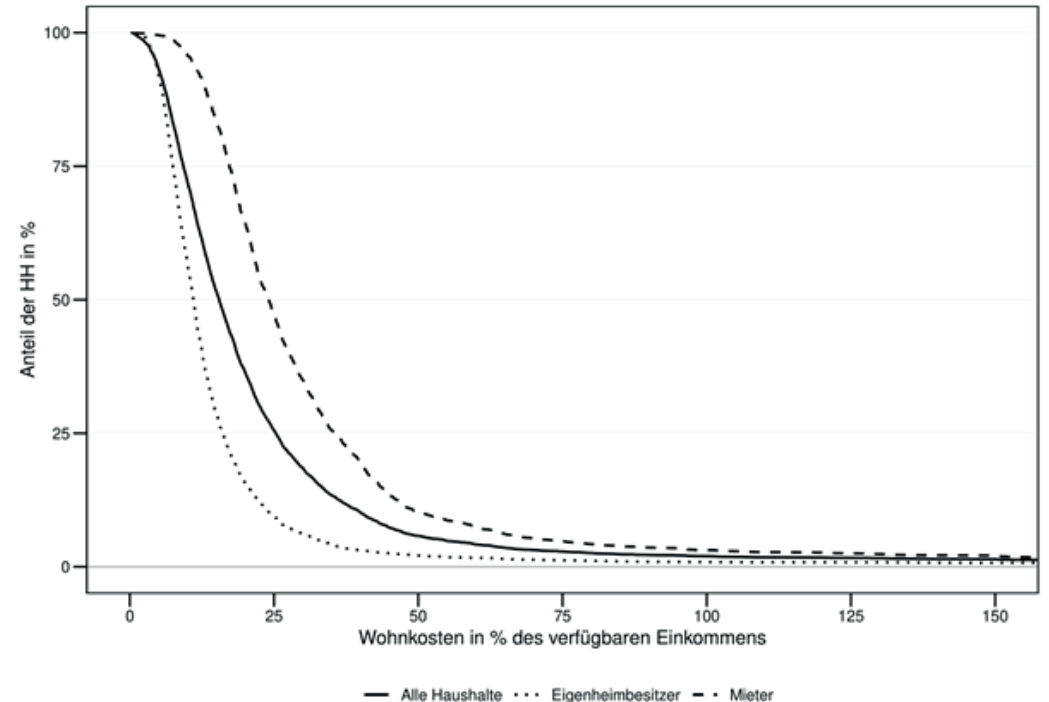

Quelle: SILC 2017

Abbildung 5: Durchschnittlicher Anteil der Wohnkosten in \% des verfügbaren Einkommens

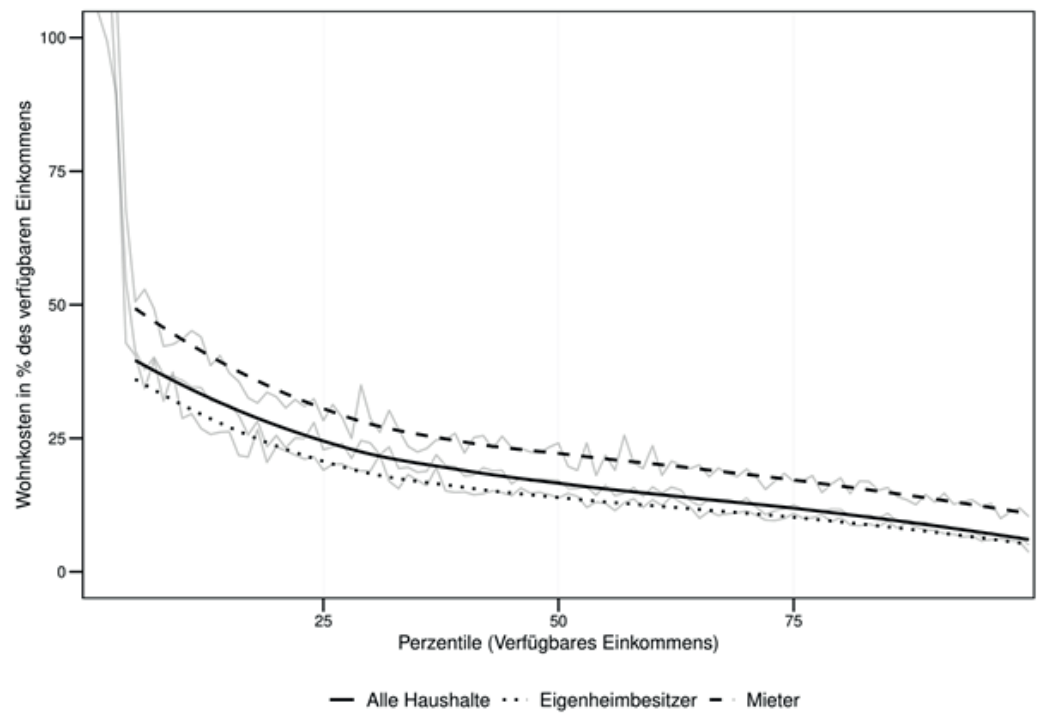

Quelle: SILC 2017. Die durchschnittlichen relativen Anteile wurden mit ,lokal linearen Polynomialregressionen (Polynom: 2. Grad, 70 Prozent Smoothing Span) geglättet. Da die relativen Wohnkosten zu Beginn aufgrund von niedrigen Einkommen sehr hoch ausfallen und stark schwanken, wurde die Glättung erst ab Perzentil 5. durchgeführt, um für den Großteil der Verteilung robuste Ergebnisse zu erhalten.

Abbildung 5 stellt die durchschnittlichen Wohnkosten in Prozent des verfügbaren Einkommens dar.

1 Da die Kurven eine deutliche Struktur, jedoch auch eine hohe Schwankung zwischen den einzelnen Perzentilsgruppen aufweisen, wurden sie zur besseren Interpretierbarkeit mit ,lokal linearen Polynomial-Regression' geglättet. Man sieht, dass am unteren Ende der Einkommensverteilung für viele Haushalte die Wohnkosten aufgrund von fehlenden Einkommen einen Anteil von über 100 Prozent ausmachen. Deshalb wurde bei den untersten 4 Perzentilen aufgrund der hohen Fluktuation von der Glättung abgesehen.
In den unteren 25 Prozent $^{2}$ betragen die durchschnittlichen Wohnkosten für alle Haushalte 31 Prozent, für EigenheimbesitzerInnen 28 Prozent und für MieterInnen 39 Prozent des verfügbaren Einkommens. Bei steigendem Einkommen sinken die relativen Wohnkosten für alle Haushalte. Für EigenheimbesitzerInnen liegen die Kosten entlang der gesamten Verteilung deutlich

2 Hier wurden wieder die ersten 4 Perzentile aufgrund der niedrigen Einkommen und den daraus resultierenden Outliern bei der relativen WKB nicht berücksichtigt. 
Abbildung 6: Anteil der Haushalte, die mehr als X Prozent ihres verfügbaren Einkommens für Wohnkosten ausgeben

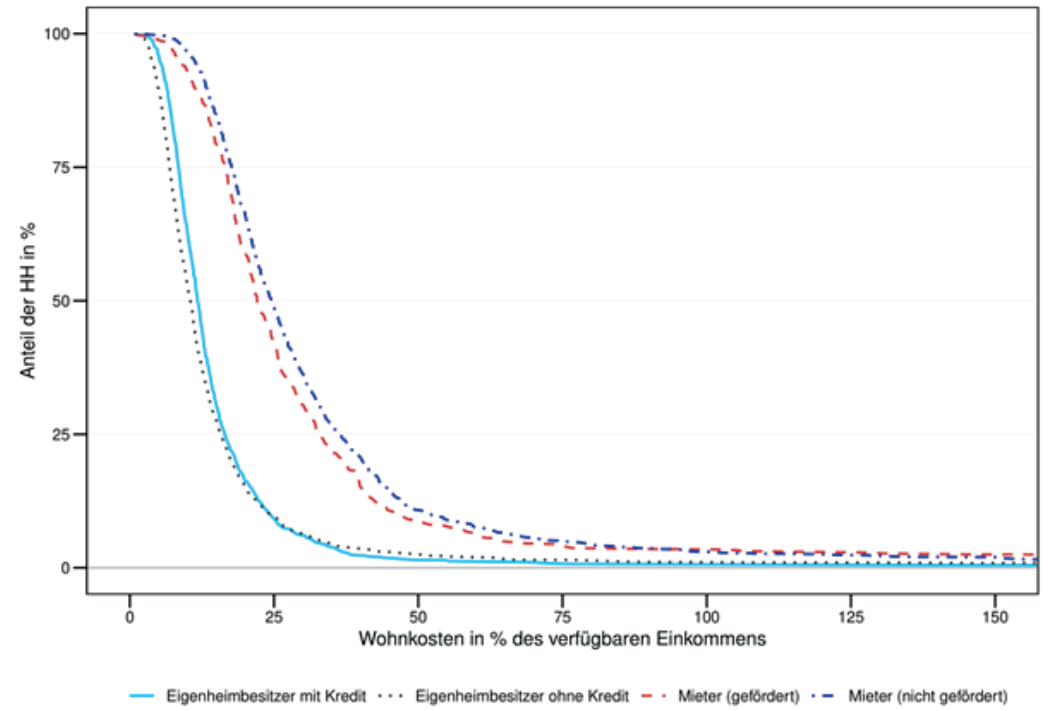

Quelle: SILC 2017

Abbildung 7: Durchschnittlicher Anteil der Wohnkosten in \% des verfügbaren Einkommens

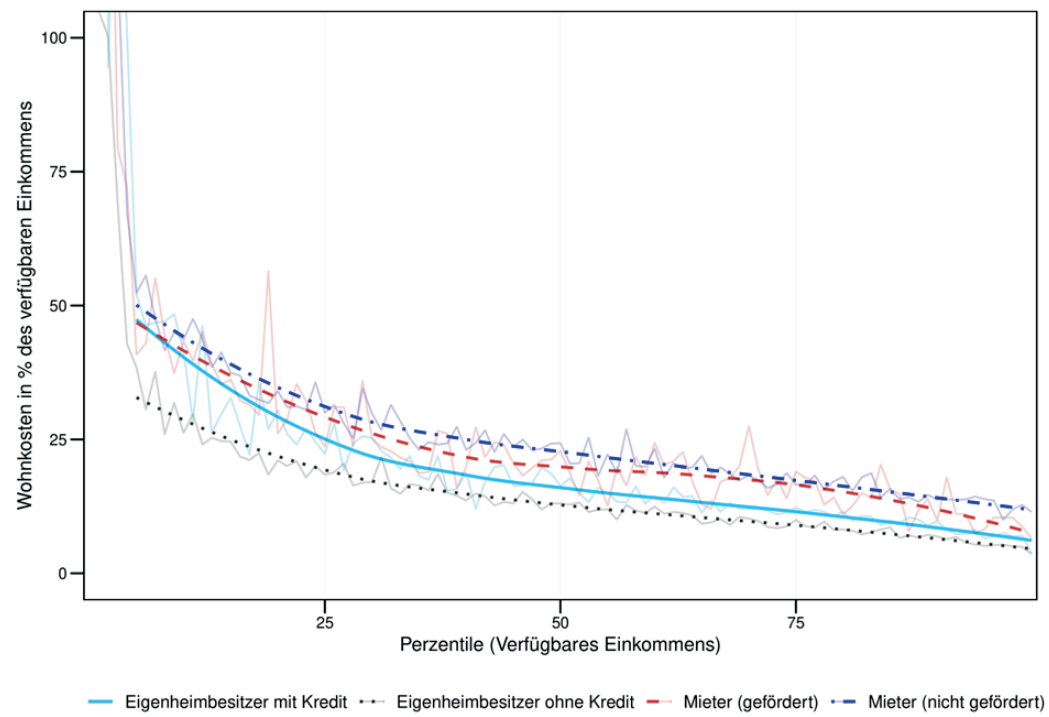

Quelle: SILC 2017. Die durchschnittlichen relativen Anteile wurden mit ,lokal linearen Polynomialregressionen (Polynom: 2. Grad, 70 \% Smoothing Span) geglättet. Da die relativen Wohnkosten zu Beginn aufgrund von niedrigen Einkommen sehr hoch ausfallen und stark schwanken, wurde die Glättung erst ab Perzentil 5. durchgeführt, um für den Großteil der Verteilung robuste Ergebnisse zu erhalten.

unter jenen der MieterInnen. Im einkommensstärksten Viertel betragen die mittleren Wohnkostenanteile für alle Haushalte 9 Prozent, für EigenheimbesitzerInnen 8 Prozent, für MieterInnen jedoch noch 14 Prozent.

In Abbildung 6 und 7 werden die Gruppen der MieterInnen und WohnungseigentümerInnen nochmals in geförderte und nicht geförderte MieterInnen bzw. EigenheimbesitzerInnen mit und ohne Hypothek eingeteilt

Während der Verlauf der EigenheimbesitzerInnen mit bzw. ohne Hypothek sehr ähnlich ausfällt (Abbil- dung 6), ist der Anteil der geförderten MieterInnen, die mehr als X Prozent ihres Einkommens für Wohnkosten aufwenden, über den Großteil des Verlaufes deutlich niedriger als jener der nicht geförderten MieterInnen. Demnach gibt ein Viertel der nicht geförderten MieterInnen mehr als 36 Prozent des verfügbaren Einkommens für Wohnkosten aus. Bei geförderten MieterInnen liegt dieser Wert bei 33 Prozent. Bei den EigenheimbesitzerInnen verwenden die 25 Prozent mit den höchsten relativen Kosten mehr als 16 Prozent (ohne Kredit) bzw. 17 Prozent (mit Kredit) ihres ver- 
Tabelle 5: Anteil der Haushalte, die mehr als X Prozent ihres verfügbaren Einkommens für Wohnkosten ausgeben

\begin{tabular}{llllll}
\hline & $10 \%$ & $20 \%$ & $30 \%$ & $40 \%$ & $50 \%$ \\
\hline Eigenheimbesitzer & 56,5 & 15,6 & 6,2 & 3,1 & 2,1 \\
davon Eigenheimbesitzer mit Kredit & 62,7 & 16,2 & 6,0 & 2,3 & 1,4 \\
davon Eigenheimbesitzer ohne Kredit & 52,6 & 15,1 & 6,2 & 3,6 & 2,5 \\
Freie Nutzer & 40,0 & 11,9 & 4,7 & 3,2 & 3,1 \\
Mieter & 95,7 & 64,4 & 35,0 & 19,3 & 10,4 \\
davon Mieter (gefördert) & 92,6 & 58,8 & 30,4 & 15,1 & 8,8 \\
davon Mieter (nicht gefördert) & 96,6 & 66,1 & 36,3 & 20,5 & 10,8 \\
Alle Haushalte & 72,0 & 36,4 & 18,5 & 10,1 & 5,8 \\
\hline
\end{tabular}

Quelle: SILC 2017

fügbaren Einkommens für Wohnkosten. Der größte Unterschied wird jedoch nach wie vor zwischen den Hauptgruppen sichtbar (MieterInnen vs. EigenheimbesitzerInnen). Die Förderungsmaßnahmen durch sozial geförderte Mietwohnungen mindern die Wohnkostenbelastung also signifikant, jedoch bleibt diese weiterhin deutlich höher als für beide Gruppen der EigenheimbesitzerInnen.

Wie in Abbildung $7 \mathrm{zu}$ sehen ist, liegen die relativen Wohnkosten für EigenheimbesitzerInnen mit und ohne Kredit entlang der gesamten Einkommensverteilung deutlich unter jenen der geförderten und nicht geförderten MieterInnen. Lediglich am unteren Ende der Verteilung ist der durchschnittliche relative Anteil der Wohnkosten für EigenheimbesitzerInnen mit Kredit in einem ähnlich hohen Bereich. Im unteren Viertel $^{3}$ beträgt die WKB bei EigentümerInnen mit Kredit 35 Prozent, bei geförderten MieterInnen 37 Prozent und bei nicht geförderten MieterInnen 40 Prozent. Im weiteren Verlauf fällt dieser Wert jedoch deutlich $\mathrm{ab}$ und bewegt sich durchgehend unter den durchschnittlichen Ausgaben der MieterInnen. Dies dürfte darauf zurückzuführen sein, dass EigentümerInnen mit Krediten zwar generell auch hohe absolute Wohnkosten haben, aber - wie in der Erläuterung zu Tabelle 3 bereits erwähnt - über ein gewisses Einkommen verfügen müssen, um einen Kredit zu erhalten, und letzterer Effekt am oberen Ende der Einkommensverteilung verhältnismäßig stärker ins Gewicht $\mathrm{zu}$ fallen scheint.

3 Hier wurden wieder die ersten 4 Perzentile aufgrund der niedrigen Einkommen und den daraus resultierenden Outliern bei der relativen WKB nicht berücksichtigt.
Die Wohnkosten von EigenheimbesitzerInnen ohne Kredit im einkommensschwächsten Viertel liegen mit 25 Prozent deutlich unter dem Niveau der anderen Gruppen. Für das einkommensstärkste Viertel liegt die durchschnittliche WKB für nicht geförderte MieterInnen bei 15 Prozent, für geförderte MieterInnen bei 12 Prozent, für EigenheimbesitzerInnen mit Kredit bei 9 Prozent und für EigenheimbesitzerInnen ohne Kredit bei 7 Prozent. Hier stellt sich die Frage, ob man Haushalte, die sich am oberen Ende der Einkommensverteilung befinden, noch durch geförderte Mieten unterstützen möchte. Andererseits wirkt eine Durchmischung von einkommensstarken und -schwächeren Haushalten in sozial geförderten Wohnanlagen oder Gemeindebauten auch einer räumlichen Segregation entgegen.

In Tabelle 5 sind zusammenfassend noch einmal die Anteile der Gruppen aufgelistet, die mehr als 10 Prozent, 20 Prozent, 30 Prozent, 40 Prozent und 50 Prozent des verfügbaren Einkommens für Wohnkosten ausgeben. Bei den EigenheimbesitzerInnen sind es beispielsweise nur 6,2 Prozent, die mehr als 30 Prozent ihres Einkommens für Wohnkosten aufwenden müssen, während es bei den MieterInnen 35,o Prozent sind. Welche soziodemografischen Gruppen besonders oft von einer hohen WKB betroffen sind, wird in Kapitel 5 näher erörtert.

\subsection{Dekomposition}

In Tabelle 6 sind verschiedene Verteilungsindikatoren vor und nach Abzug der Wohnkosten aufgelistet. Der Gini steigt dabei von 0,35 auf 0,40 an. Der beträchtliche Anstieg indiziert eine deutliche Erhöhung der Ungleichheit und den starken Einfluss der Wohnkosten auf die ökonomischen Lebensrealitäten.
MOMENTUM QUARTERLY "IIIIIIIIIIIIIIIIIII 171 
Tabelle 6: Ungleichheitsmaße für das verfügbare Einkommen (vor und nach Abzug der Wohnkosten)

\begin{tabular}{llccccc}
\hline & \multicolumn{3}{c}{ Verfügbares Einkommen } & \multicolumn{2}{c}{ Verfügbares Einkommen nach Abzug der Wohnkosten } \\
\hline & Wert & Within & Between & Wert & Within & Between \\
Gini & 0,35 & & & 0,40 & & \\
Theil & 0,22 & $88 \%$ & $12 \%$ & 0,27 & $85 \%$ & $15 \%$ \\
P90/P50 & 2,14 & & & 2,37 & & \\
P90/P10 & 5,44 & & & 7,93 & & \\
\hline
\end{tabular}

Quelle: SILC 2017

Der Theil-Index ist ein Verteilungsmaß aus der Klasse der generalisierten Entropie-Indizes und ermöglicht eine Dekomposition, bei der die Verteilungswirkung in zwei Teile zerlegt werden kann. Nämlich in jenen, der sich aufgrund der Ungleichheit zwischen den Gruppen ergibt, und jenen, der auf die Ungleichheit innerhalb der einzelnen Gruppen zurückzuführen ist. In dieser Anwendung können somit zwei unterschiedliche Arten von Ungleichheit unterschieden werden: Die Ungleichheit, die sich aufgrund der verschiedenen Einkommen zwischen MieterInnen und EigentümerInnen ergibt, sowie die Ungleichheit in diesen Gruppen selbst.

Der Index steigt von 0,22 auf 0,27, wobei sich der Anteil, der sich aus der Ungleichheit zwischen den Gruppen ergibt, von 12 Prozent auf 15 Prozent erhöht. Somit wird auch hier ersichtlich, dass sich nicht nur die gesamte Ungleichheit durch die Wohnkosten erhöht, sondern vor allem die Ungleichheit zwischen den Gruppen.

Sowohl bei dem $\mathrm{P}_{90} / \mathrm{P}_{50}$ als auch beim $\mathrm{P}_{90} / \mathrm{P}_{10}$ Ratio $^{4}$ ist ein deutlicher Anstieg zu erkennen. Die starke Erhöhung des P9o/P10 Ratios von 5,44 auf 7,93 indiziert wiederum, dass die WKB vor allem am unteren Ende der Einkommensverteilung eine stärkere Belastung darstellt. Die Betrachtung der Indikatoren zeigt auf, dass die WKB wesentlich ungleicher verteilt ist als die verfügbaren Einkommen selbst. Da die gemessene Ungleichheit bei allen Verteilungsmaßen stark ansteigt, wird wieder ersichtlich, dass die WKB für Haushalte mit geringerem Einkommen höher ist. Folglich können potenzielle Maßnahmen zur Entlastung der Wohnkosten in den niedrigen Einkommensgruppen im großen Ausmaß zur Reduktion der ungleichen Lebensverhältnisse beitragen.

4 Bei diesen Indikatoren handelt es sich um das relative Einkommensverhältnis der Perzentilsgrenzen.

\section{Soziodemografische Faktoren}

Während in den vorangegangenen Kapiteln die Segregation und die WKB anhand des verfügbaren Einkommens aufgezeigt wurde, wird in diesem Kapitel untersucht, welche soziodemografischen Faktoren mit einer hohen WKB assoziiert sind. Als Ausgangspunkt dient dazu Abbildung 8, in der die Haushalte entlang des relativen Anteils der Wohnkosten am verfügbaren Einkommen aufgereiht sind. Wieder ist klar zu sehen, dass es fast ausschließlich EigentümerInnen und Freie NutzerInnen sind, die nur eine verhältnismäßig geringe Belastung aufweisen. Auf der anderen Seite sind es großteils MieterInnen, die von einer hohen WKB betroffen sind.

In der Literatur ist die gängige Grenze, $a b$ der man von einer kritischen WKB spricht, bei 30 Prozent angesetzt. Um ein etwas detaillierteres Bild zu erhalten, werden in diesem Bericht mehrere Belastungsgrenzen herangezogen. Die vertikalen Linien in Abbildung 8 indizieren, welche Haushalte mehr als 30 Prozent, 40 Prozent bzw. 50 Prozent ihres verfügbaren Einkommens für Wohnkosten ausgeben. Bei diesen Haushalten handelt es sich um 18,5 Prozent, 10,0 Prozent bzw. 5,8 Prozent der gesamten Haushalte (siehe Tabelle 5).

Um zu determinieren, welche Faktoren mit einer hohen WKB assoziiert sind, verwenden wir sogenannte ,Recentered Influence Function Regressions (RIF-Regressionen), die es erlauben, die marginalen Effekte der Kovariaten auf eine Verteilungsstatistik zu regressieren. Diese Analyse erlaubt es zu bestimmen, welche einzelnen Faktoren eines Haushaltes die Wahrscheinlichkeit erhöhen, in dem Bereich über der WKB-Grenze zu liegen, da gleichzeitig für alle anderen Faktoren kontrolliert wird.

Wenn man also beispielsweise lediglich die Mittelwerte nach Bildungsabschluss vergleichen würde, wären diese Gruppen gleichzeitig wieder mit anderen 
Abbildung 8: Wohntypen entlang der Verteilung der Wohnkosten als Anteil am verfügbaren Einkommen (in Prozent)

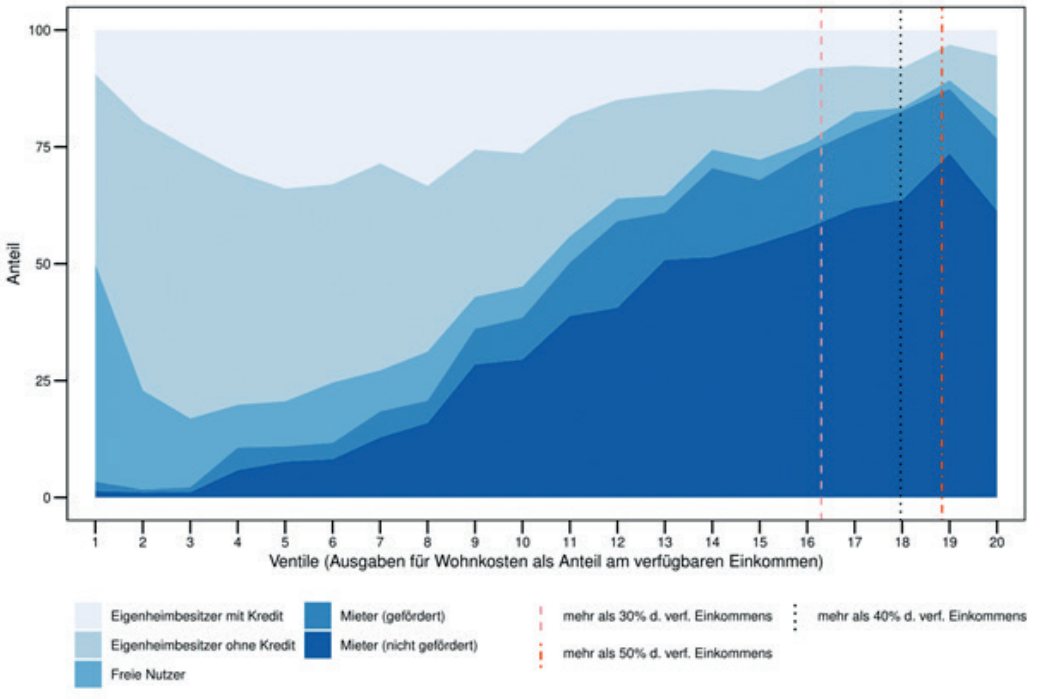

Quelle: SILC 2017.

Eigenschaften, wie einem höheren/niedrigeren Einkommen, Alter oder Wohnverhältnis, korreliert und somit wäre die Interpretation des marginalen Effektes nicht möglich.

Tabelle 7 zeigt die Ergebnisse der statistischen Schätzungen. Neben den RIF-Regressionen für die WKB-Grenzen am oberen Ende der Verteilung wurde auch eine OLS-Regression geschätzt, die den durchschnittlichen Effekt beschreibt. Zusätzlich wurde auch eine Median-Regression durchgeführt, die den Effekt der soziodemografischen Faktoren in der Mitte der Verteilung misst, jedoch robuster gegen Ausreißer ist als die OLS-Regression. ${ }^{5}$

Aufgrund der Auswahl der Dummy-Variablen handelt es sich bei der Baseline (Intercept) um einen Haushalt, der Eigenheimbesitzer mit Kredit ist, in dem niemand der Haushaltsmitglieder einen höheren Bildungsabschluss (AHS/BHS oder Universität) hat, der in einer Gegend mit mittlerer Bevölkerungsdichte wohnt, in dem das durchschnittliche Alter der Erwachsenen unter 35 liegt, der keine Kinder im Haushalt hat und kein Single-Haushalt ist.

5 Da die abhängige Variable ein Verhältniswert ist kann dieser Wert zum einen nicht definiert sein, wenn kein Einkommen vorhanden ist, oder aber auch sehr hoch, wenn das Einkommen nur sehr gering ist. Die OLS-Regression reagiert auf diese Werte im Gegensatz zu den RIF-Regressionen äußerst sensibel. Deshalb wurden Werte größer als 100 Prozent einheitlich auf 100 Prozent gesetzt.
Die OLS- und Median-Regression zeigen großteils einheitliche Ergebnisse (siehe Tabelle 7). MieterInnen sind stark mit einer hohen WKB assoziiert, wobei die Belastung für nicht geförderte MieterInnen strukturell höher ist. Ob man als EigenheimbesitzerIn einen offenen Kredit hat, spielt bei der Median-Regression nur eine kleine Rolle, während dieser Effekt bei der OLSSchätzung nicht signifikant ist. Hingegen sind Freie NutzerInnen deutlich weniger von hohen Wohnkosten betroffen. Im Bereich der Ausbildung ist ein Abschluss einer AHS/BHS nach OLS mit einer niedrigeren WKB assoziiert, der Abschluss einer Universität ist allerdings nicht signifikant korreliert. Der Urbanisierungsgrad, gemessen an der Bevölkerungsdichte, zeigt eine signifikante Korrelation mit einer niedrigeren WKB für Gebiete mit einer niedrigeren Bevölkerungsdichte. Bezüglich der Altersstruktur zeigt sich, dass Haushalte, in denen das durchschnittliche Alter der Erwachsenen über 65 Jahren liegt, deutlich weniger von hohen Wohnkosten betroffen sind. Bei der OLS-Regression ist dieser Effekt auch für die Gruppe der 35- bis 65-Jährigen zu sehen. Dies zeigt, dass die jüngere Generation (< 35 Jahre) deutlich stärker von einer hohen WKB betroffen ist. Kinder weisen keinen signifikanten Effekt auf, während Single-Haushalte mit höheren WKB assoziiert werden.

Bei der Analyse der sozioökonomischen Determinanten für Haushalte, die besonders stark von hohen Wohnkosten betroffen sind, indiziert der Regressionsoutput für die 30-Prozent-WKB-Grenze, also jene 
Tabelle 7: Regressionsoutput für soziodemografische Faktoren, Quantilsgrenze: mehr als X Prozent des verfügbaren Einkommens werden für Wohnkosten ausgegeben

\begin{tabular}{|c|c|c|c|c|c|c|c|c|c|c|}
\hline \multirow[b]{3}{*}{ Intercept } & \multirow{2}{*}{ OLS } & & \multicolumn{8}{|c|}{ RIF-Regressionen } \\
\hline & & & MEDIAN & & $>30 \%$ & & $>40 \%$ & & $>50 \%$ & \\
\hline & 29,255 & 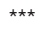 & 19,642 & 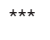 & 40,322 & 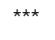 & 54,393 & *** & 76,856 & *** \\
\hline Eigenheimbesitzer ohne Kredit & $-1,090$ & & $-1,660$ & 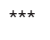 & $-0,337$ & & 2,322 & & 6,150 & * \\
\hline Freie Nutzer & $-7,647$ & 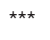 & $-7,416$ & 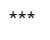 & $-7,987$ & 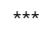 & $-5,176$ & & $-2,119$ & \\
\hline Mieter (gefo "rdert) & 6,980 & 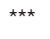 & 9,951 & 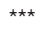 & 12,417 & 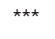 & 11,400 & $\star *$ & 10,698 & \\
\hline Mieter (nicht gefo" rdert) & 9,440 & 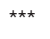 & 11,208 & 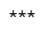 & 17,285 & 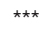 & 19,930 & 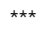 & 19,428 & *** \\
\hline Nettoeinkommen & $-0,003$ & 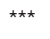 & $-0,002$ & 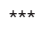 & $-0,004$ & 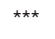 & $-0,005$ & 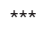 & -0.009 & 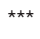 \\
\hline AHS/BHS & $-2,772$ & 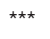 & $-1,038$ & & $-4,868$ & * & $-7,155$ & * & $-10,654$ & \\
\hline Universita"t & $-0,588$ & & $-1,084$ & & $-1,062$ & & 0,030 & & 6,155 & \\
\hline Niedrige Bevo" lkerungsdichte & $-1,228$ & $\star \star$ & $-1,304$ & $\star \star$ & $-1,734$ & & $-1,576$ & & $-2,905$ & \\
\hline Hohe Bevo" lkerungsdichte & 0,265 & & 0,163 & & $-0,373$ & & $-2,141$ & & 6,101 & \\
\hline 35-65 Jahre & $-1,855$ & 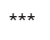 & $-1,104$ & $\star \star$ & $-1,841$ & & $-3,572$ & & $-8,499$ & \\
\hline$>65$ Jahre & $-2,832$ & 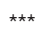 & $-0,105$ & & $-4,872$ & 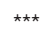 & $-8,486$ & $\star \star$ & $-21,629$ & $\star \star \star$ \\
\hline Kinder im Haushalt & 0,787 & & 0,046 & & 2,451 & & 2,594 & & 4,221 & \\
\hline Single & 2,935 & 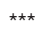 & 2,582 & 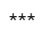 & 6,279 & 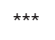 & 4,695 & * & 10,384 & \\
\hline Kinder*Single & 10,352 & 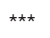 & $-3,584$ & & 11,479 & & 38,292 & $\star \star$ & 109,946 & ** \\
\hline
\end{tabular}

Quelle: SILC 2017

Stelle, an der 18,5 Prozent der Haushalte mehr und 81,5 Prozent der Haushalte weniger als 30 Prozent für Wohnkosten ausgeben, für MieterInnen wieder eine höhere WKB, wobei der Koeffizient für geförderte MieterInnen bei den höheren Grenzen für die WKB zurückgeht und an der 50-Prozent-WKB-Grenze nicht mehr signifikant ist. Für nicht geförderte MieterInnen erhöht sich der Koeffizient leicht und bleibt für alle Grenzen hochsignifikant. Haushalte, die in einer nicht geförderten Mietwohnung leben, sind auch bei dieser Analyse wieder mit einer sehr hohen WKB assoziiert. Ein AHS/BHS-Bildungsabschluss ist ebenfalls wieder mit einer reduzierten WKB korreliert, auch wenn der Koeffizient vergleichsweise gering ausfällt. Die Bevölkerungsdichte weist keine signifikanten Effekte mehr auf, während die Gruppe der über 65-Jährigen strukturell weniger von hohen Wohnkosten betroffen ist. Der höhere Belastungseffekt für Singles ist an der 30-Prozent- und 40-Prozent-WKB-Grenze noch signifikant, während der Interaktionseffekt für AlleinerzieherInnen erst ab der 40-Prozent-WKB-Grenze signifikant ist. Der marginale Effekt dieses Interaktionskoeffizienten ist mit Abstand der stärkste in der Analyse. Alleinerziehende sind also besonders stark von einer hohen WKB betroffen, wobei der Koeffizient $(109,0)$ an der 50-Prozent-WKB-Grenze fünfmal so hoch wie jener für nicht geförderte MieterInnen $(19,4)$ ist.

\section{Subjektive vs. objektive Wohnkostenbelastung}

In diesem Abschnitt werden die diskutierten Ergebnisse mit der subjektiven WKB verglichen, die ebenfalls im Zuge des SILC erhoben wird. Bei der subjektiven WKB wird erfragt, ob die Wohnkosten keine, eine mittlere oder eine hohe Belastung darstellen. Für die weitere Analyse wird nur eine hohe subjektive WKB (11,8 Prozent aller Haushalte) berücksichtigt, da nur wenige Haushalte angeben, die Wohnkosten als keinerlei finanzielle Belastung zu empfinden.

Um die objektive Belastung, die wie bisher als Anteil der Wohnkosten am verfügbaren Einkommen angegeben wird, mit der subjektiven vergleichbar $\mathrm{zu}$ machen, definieren wir dieselbe Anzahl an Haushalten (11,8 Prozent) als Haushalte mit einer objektiven WKB. Das sind jene Haushalte, die mehr als ca. 37 Prozent ihres verfügbaren Einkommens für Wohnkosten ausgeben. In einem ersten Schritt wird visualisiert, ob die einzelnen Wohngruppen überproportional von Wohnkosten belastet sind. Dazu wird anhand von Tabelle 8 die Anzahl der Wohngruppen mit objektiver WKB zur gesamten Anzahl der Wohn- 
Tabelle 8: Anteil der Wohngruppen (in \%) allgemein vs. Anteil der Wohngruppen mit starker objektiver Wohnkostenbelastung

\begin{tabular}{lccc}
\hline \multicolumn{1}{c}{ Wohntyp } & Insgesamt & Starke obj. WKB & Ratio \\
\hline Eigenheimbesitzer mit Kredit & 18,54 & 4,63 & 0,25 \\
Eigenheimbesitzer ohne Kredit & 29,24 & 9,51 & 0,33 \\
Freie Nutzer & 9,10 & 2,65 & 0,29 \\
Mieter (gefördert) & 9,81 & 16,00 & 1,63 \\
Mieter (nicht gefördert) & 33,31 & 67,22 & 2,02 \\
& 100,0 & 100,0 & \\
\hline
\end{tabular}

Quelle: SILC 2017

Abbildung 9: Verhältnis des durchschnittlichen Anteils der Wohngruppen zu Wohngruppen mit starker obj. Wohnkostenbelastung

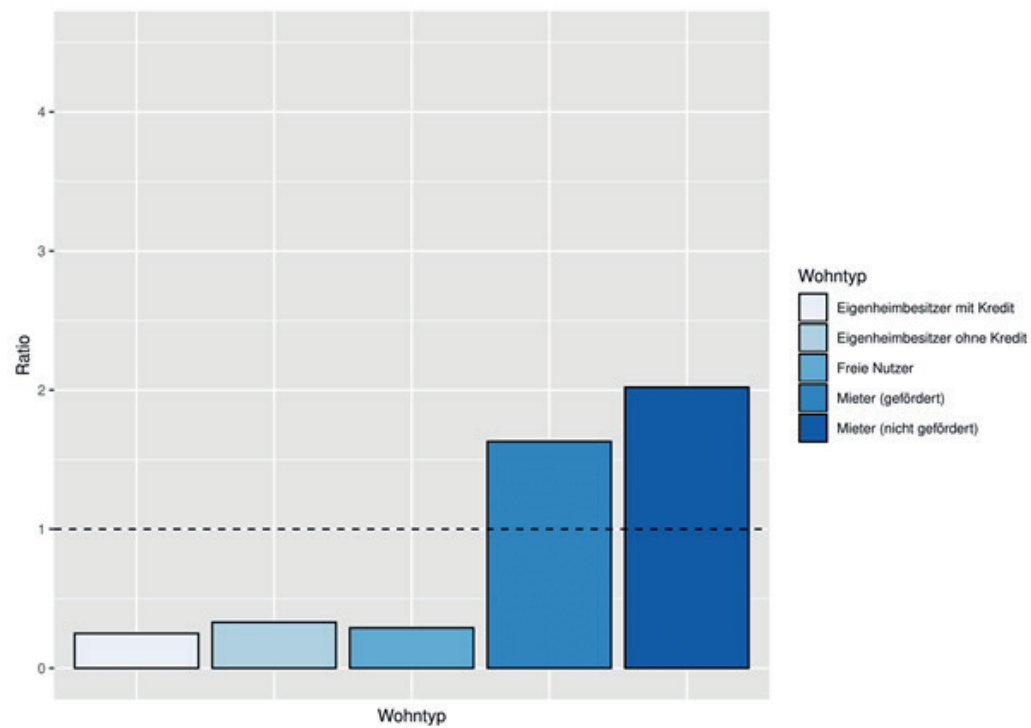

Quelle: SILC 2017. Ein Ratio von 1 würde bedeuten, dass die jeweilige Gruppe weder überdurchschnittlich noch unterdurchschnittlich durch Wohnkosten belastet ist. Ein Wert größer 1 indiziert eine überdurchschnittlich hohe WKB und vice versa.

gruppe in Relation gesetzt. Ein Ratio von 1 würde also bedeuten, dass der Gesamtanteil einer Wohngruppe genauso hoch ausfällt wie deren Anteil in der Gruppe der Haushalte mit objektiver WKB, und somit keine über- oder unterproportionale WKB gegeben ist.

Dies ist allerdings nicht der Fall, wie in Abbildung $9 \mathrm{zu}$ sehen ist. So sind EigenheimbesitzerInnen mit Kredit nur 0,25-mal so oft in der Gruppe mit objektiver WKB zu finden wie in der Grundgesamtheit. Bei EigenheimbesitzerInnen ohne Kredit und Freien NutzerInnen beträgt dieser Wert ebenfalls nur o,33 bzw. 0,29. Auf der anderen Seite sind geförderte MieterInnen 1,63-mal so oft und nicht geförderte MieterInnen sogar 2,02-mal so oft in dieser Gruppe anzutreffen. Wieder zeigt sich hier die entlastende Wirkung der wohnpolitischen Maßnahmen, die eine besonders hohe
WKB für geförderte MieterInnen im Vergleich zu nicht geförderten MieterInnen minimiert, aber dennoch um ein Vielfaches stärker ausgeprägt ist als für EigenheimbesitzerInnen.

Würden alle Haushalte den oben beschriebenen Sachverhalt, also die überproportional hohe Belastung von MieterInnen, korrekt einschätzen, würde das Ratio von subjektiver und objektiver WKB bei 1 liegen. Dies trifft allerdings nicht zu, da vor allem EigenheimbesitzerInnen mit Kredit ein verzerrtes Bild ihrer relativen WKB haben. Sie geben an, überproportional durch hohe Wohnkosten belastet zu sein, und machen damit 20,44 Prozent aller Haushalte aus, die angeben, von starken WKB betroffen zu sein, während deren Anteil nach objektivem Maß nur 4,63 Prozent beträgt (siehe Tabelle 9). 
Tabelle 9: Anteil der Wohngruppen (in \%) mit starker objektiver Wohnkostenbelastung vs. Anteil der Wohngruppen mit starker subjektiver Wohnkostenbelastung

\begin{tabular}{llll}
\hline \multicolumn{1}{c}{ Wohntyp } & \multicolumn{1}{c}{ Starke subjektive WKB } & Starke objektive WKB & Ratio \\
\hline Eigenheimbesitzer mit Kredit & 20,44 & 4,63 & 4,42 \\
Eigenheimbesitzer ohne Kredit & 12,55 & 9,51 & 1,32 \\
Freie Nutzer & 4,31 & 2,65 & 1,63 \\
Mieter (gefördert) & 7,95 & 16,00 & 0,50 \\
Mieter (nicht gefördert) & 54,74 & 67,22 & 0,81 \\
& 100,0 & 100,0 & \\
\hline
\end{tabular}

Quelle: SILC 2017

Abbildung 10: Verhältnis des durchschnittlichen Anteils der Wohngruppen zu Wohngruppen mit starker obj. Wohnkostenbelastung

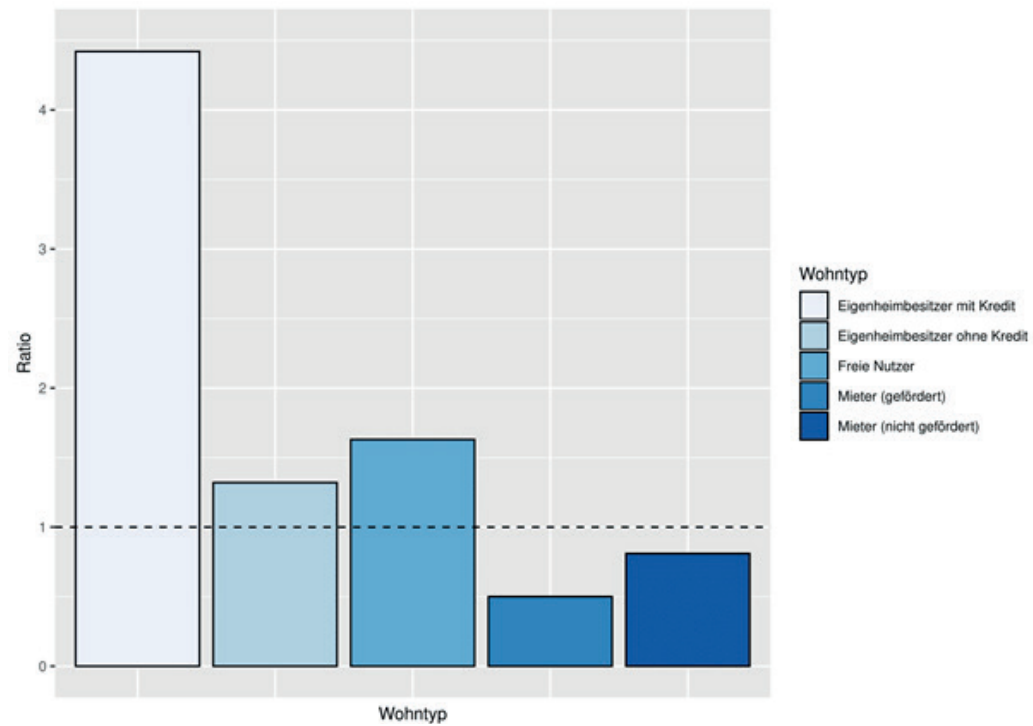

Quelle: SILC 2017. Ein Ratio von 1 würde bedeuten, dass die subjektive Einschätzung bezüglich der WKB der objektiven entspricht. Ein Wert größer 1 indiziert, dass die subjektive WKB im Vergleich zur tatsächlichen Belastung zu hoch eingeschätzt wird und vice versa.

Somit denken EigenheimbesitzerInnen mit Kredit 4,42-mal so oft von einer starken WKB betroffen zu sein, als es tatsächlich der Fall ist. Auch die Gruppe der EigenheimbesitzerInnen ohne Kredit und der Freien NutzerInnen überschätzt die Betroffenheit mit einem Faktor von 1,32 und 1,63. Bei den MieterInnen verhält es sich umgekehrt, da diese vergleichsweise weniger oft angeben, eine hohe Belastung durch Wohnkosten zu empfinden. So machen die geförderten MieterInnen 7,95 Prozent und die nicht geförderten MieterInnen 54,74 Prozent aller Haushalte aus, die angeben, von hohen WKB betroffen zu sein, während sie nach objektivem Maßstab 16,0 Prozent bzw. 67,22 Prozent ausmachen. Folglich unterschätzen die geförderten MieterInnen ihre WKB mit einem Faktor von 0,50 und die nicht geförderten MieterInnen mit o,81.

\section{Conclusio}

Die vorliegende Studie hat die charakteristischen Unterschiede zwischen WohnungseigentümerInnen und MieterInnen deutlich gemacht. Generell zeigen sich zwischen diesen beiden Gruppen deutliche Einkommensunterschiede. Das durchschnittlich verfügbare Monatseinkommen eines Mieterhaushaltes beträgt 2.90o Euro gegenüber 4.40o Euro eines Eigentümerhaushaltes. Generell ändert sich die Relation von WohnungseigentümerInnen zu MieterInnen entlang der Einkommensverteilung stark: Während im unteren Einkommensdrittel der Anteil der MieterInnen rund 60 Prozent ausmacht, fällt dieser im einkommensstärksten Drittel auf unter 30 Prozent. Demgegenüber steigt der Anteil an WohnungseigentümerInnen von rund 20 Prozent bei den untersten Einkommen auf knapp 80 Prozent im obersten Dezil der Einkommensverteilung. 
Darüber hinaus unterscheiden sich jedoch nicht nur die Einkommen zwischen MieterInnen und EigentümerInnen gravierend, sondern auch die Wohnkosten selbst. Wohnkosten spielen für Haushalte, die ihren Hauptwohnsitz mieten, eine wesentlich größere Rolle; und dies nicht nur relativ, sondern auch absolut. Die monatlichen Wohnkosten für EigentümerInnen betragen im Durchschnitt 448 Euro, während diese für MieterInnen mit 646 Euro um 40 Prozent höher ausfallen.

Im Weiteren wurde untersucht, welche Haushalte von einer hohen Wohnkostenbelastung betroffen sind. Unter hoher Wohnkostenbelastung wird hier ein Schwellenwert von 40 Prozent definiert; d.h. jene Haushalte, die mehr als 40 Prozent ihres verfügbaren Einkommens für Wohnkosten ausgeben müssen (vgl. Tabelle 4).

Bei dieser Definition sind insgesamt 10,1 Prozent aller Haushalte mit einer hohen Wohnkostenbelastung konfrontiert. Bei WohnungseigentümerInnen sind dies jedoch nur 3,1 Prozent, während dieser Anteil bei MieterInnen mit 19,3 Prozent ein Vielfaches beträgt. Es zeigt sich somit, dass eine hohe Wohnkostenbelastung nahezu ausschließlich bei MieterInnen anfällt. Interessante Unterschiede zeigen sich auch zwischen WohnungseigentümerInnen ohne und mit Kredit: Während bei der ersten Gruppe eine hohe Wohnkostenbelastung bei 3,6 Prozent aller Haushalte auftritt, ist dieser Anteil bei Haushalten mit Krediten nur 2,3 Prozent. Dieser überraschende Unterschied kann jedoch damit erklärt werden, dass WohnungseigentümerInnen mit Kredit im Mittel über ein deutlich höheres Einkommen verfügen als jene ohne Kredit.

Selbst wenn man Tilgungszahlungen, die eigentlich ein Investment darstellen und nicht zu den Kosten gerechnet werden, zu den Wohnkosten hinzuzählen würde, wäre die relative Wohnkostenbelastung der EigentümerInnen mit Krediten immer noch geringer als jene der MieterInnen. Auch zwischen den beiden Gruppen von MieterInnen zeigen sich interessante Unterschiede. Während eine hohe Wohnkostenbelastung bei geförderten MieterInnen in nur 15,1 Prozent aller Fälle auftritt, ist dies bei nicht geförderten MieterInnen bei 20,5 Prozent der Fall. Es zeigt sich, dass geförderte Mieten die Wohnkostenbelastung deutlich senken.

Auch die ökonometrische Analyse nach soziodemografischen Faktoren bestätigt die hohen Aufwendungen von MieterInnen und zeigt zudem, dass vor allem junge Menschen, Single-Haushalte und in besonders hohem Ausmaß jene allein lebenden Personen, die auch Kinder im Haushalt haben, eine hohe Wohnkostenbelastung aufweisen.

Konträr zu diesem empirischen Befund empfinden EigentümerInnen mit Kredit überdurchschnittlich häufig eine hohe Wohnkostenbelastung. Obwohl diese Haushalte nicht nur deutlich höhere Einkommen aufweisen, sondern im Durchschnitt auch weniger von ihrem Einkommen für Wohnkosten ausgeben müssen, empfinden diese Haushalte eine besonders hohe Wohnkostenbelastung. Demgegenüber unterschätzen MieterInnen ihre tatsächliche Wohnkostenbelastung. Diese Gegensätze zwischen subjektiv empfundener und objektiv gemessener Wohnkostenbelastung zwischen WohnungseigentümerInnen und MieterInnen sind ein interessantes Beispiel von verzerrten Interessen mit möglicherweise weitreichenden Auswirkungen für die Bildung von individuellen Präferenzen sowie politischen Entscheidungen.

Wirtschaftspolitische Maßnahmen, die es zum Ziel haben, die Wohnkostenbelastung für jene Haushalte zu senken, die davon am stärksten betroffen sind, sollten vor allem MieterInnen entlasten. Mögliche Maßnahmen wären die Ausweitung des sozialen Wohnbaus und der Gemeindebauwohnungen (Bacher/Tamesberger 2019; Tamesberger/Bacher/Stöger 2019) oder die Streichung der Mehrwertsteuer auf private Mieten, wie dies beispielsweise in Deutschland der Fall ist (Lebuhn et al. 2017). Demgegenüber können derzeit Aufwendungen für Wohnraumschaffung von EigentümerInnen als Sonderausgaben geltend gemacht werden und mindern somit deren Steuerbelastung. Zusätzlich gibt es auf Landesebene Eigenheimförderungen, die in der Regel in der Form eines günstigen Darlehens erfolgen. Auch diese Maßnahmen tragen dazu bei, die Einkommensungleichheit nach Abzug der Wohnkosten zu verstärken, da sie vor allem BesserverdienerInnen zugutekommen und die Wohnkostenbelastung tendenziell am oberen Ende der Einkommensverteilung vermindern.

Wenngleich die hier vorgeschlagenen Maßnahmen auch noch eine nähere Spezifizierung benötigen, so ist klar, dass ein sozialer Ausgleich in der Wohnpolitik vor allem über eine stärkere Förderung sowie einen Ausbau des geförderten Mietwohnsektors erfolgreich sein wird. Eine Förderung von Wohneigentumsbildung hingegen setzt immer ein Minimum an Vermögen sowie mittleres oder hohes Einkommen voraus. Wie unsere Datenanalyse zeigt, würde eine derartige Förderpolitik somit an der Zielgruppe ,Haushalte mit hoher Wohnkostenbelastung ' nahezu vollkommen vorbeigehen.
IMOMENTUM QUARTERLY IIIIIIIIIIIIIIIIII 177 


\section{Literatur}

Amann, W./Mundt, A. (2019): Rahmenbedingungen und Handlungsoptionen für qualitätsvolles, dauerhaftes, leistbares und inklusives Wohnen. Wien: Bundesministerium für Arbeit, Soziales, Gesundheit und Konsumentenschutz.

Bacher, J./Tamesberger, D. (2019): Wie kann günstiger Wohnraum definiert werden und was sind sinnvolle Maßnahmen, um diesen zu erweitern? Momentum Quarterly, 8 (4), 183-197.

Beer, C./Wagner, K. (2012): Wohnkostenbelastung der österreichischen Haushalte. Geldpolitik und Wirtschaft, 4, 82-94.

Beer, C./Wagner, K. (2017): Household's housing expenditure in Austria, Germany and Italy. Monetary Policy and the Economy, Q4, 48-61.

Canberra Group (2011): Handbook on household income statistics. Second edition. Genf: United Nations.

Fessler, P./Rehm, M./Tockner, L. (2016): The impact of housing non-cash income on the household income distribution in Austria. Urban Studies, 53 (13), 2849-2866.

Fessler, P./Schürz, M. (2010): Informationen zum „kleinen Häuselbauer“. Wirtschaft und Gesellschaft, 36 (2), 181.

Figari, F./Paulus, A./Sutherland, H./Tsakloglou, P./Verbist, G./Zantomio, F. (2017): Removing homeownership bias in taxation: The distributional effects of including net imputed rent in taxable income. Fiscal Studies, 38 (4), 525-557.

Firpo, S./Fortin, N./Lemieux, T. (2009): Unconditional quantile regressions. Econometrica, 77 (3), 953-973.

International Labour Organisation (2003): Resolution concerning household income and expenditure statistics. Discussion paper, ILO.

Kunnert, A./Baumgartner, J. (2012a): Instrumente und Wirkungen der österreichischen Wohnungspolitik. Wien: Österreichisches Institut für Wirtschaftsforschung.

Lebuhn, H./Holm, A./Junker, S./Neitzel, K. (2017): Wohnverhältnisse in Deutschland - eine Analyse der sozialen Lage in 77 Großstädten. https://www.boeckler.de/pdf_ fof/99313.pdf [28.09.2020]

Organization for Economic Cooperation and Development (2013): Resolution concerning household income and expenditure statistics. Discussion paper, OECD.

Statistik Austria (2020): Wohnen 2019. Mikrozensus - Wohnungserhebung und EU-SILC. Wien: Statistik Austria.

Stone, M. E. (2006): What is housing affordability? The case for the residual income approach. Housing Policy Debate, 17 (1), 151-184.

Tamesberger, D./Bacher, J./Stöger, H. (2019): Die Wirkung des sozialen Wohnbaus in Österreich. Ein Bundesländervergleich. WISO, 42 (4), 29-58.

Wagner, K. (2011): Haben Mieter oder Eigentümer höhere Wohnkosten? Wirtschaft und Gesellschaft, 37 (1), 139-148. 